TITLE:

\title{
Inductive Inference of Logic Programs Based on Algebraic Semantics
}

$\operatorname{AUTHOR}(S):$

SAKAKIBARA, Yasubumi

\section{CITATION:}

SAKAKIBARA, Yasubumi. Inductive Inference of Logic Programs Based on Algebraic Semantics. 数理解析研究所講究録 1988, 655: 64-83

ISSUE DATE:

1988-04

URL:

http://hdl.handle.net/2433/100513

RIGHT: 


\title{
Inductive Inference of Logic Programs Based on Algebraic Semantics
}

\author{
Yasubumi SAKAKIBARA（榊原 康文） \\ IIAS-SIS, FUJITSU LIMITED
}

\begin{abstract}
In this paper we will present a new inductive inference algorithm for a class of logic programs, called linear monadic logic programs, in the sense that it is different from the Shapiro's Model Inference System. It is known that a set of trees is rational if and only if it is computed by a linear monadic logic program, and that the rational set of trees is recognized by tree automata. On the other hand, several efficient inference algorithms for finite automata are developed. We will extend them to an inference algorithm for tree automata and use it to get an efficient inductive inference algorithm for linear monadic logic programs. The correctness, time complexity and several comparisons of the algorithm with the Model Inference System will be shown.
\end{abstract}

\section{Introduction}

The study of inductive inference of logic programs was initially and mostly done by E.Shapiro and his work is known as the Model Inference System [5,6]. He devises a program that infers first order sentences (Horn clauses) from examples of their logical consequences. The target of the inference is an Herbrand model. Thus Shapiro's algorithm (especially the diagnosis algorithm) deeply depends on the theory of predicate logic and logic programming. In the theory of logic programming, the least model $\cap \mathrm{M}(\mathrm{LP})$ of a logic program LP is taken as the mathematical semantics, called model-theoretic semantics, for it. This semantics provides the denotation of a predicate symbol $P$ in a logic program LP :

$$
\mathrm{D}(\mathrm{P})=\left\{\left(\mathrm{t}_{1}, \ldots, \mathrm{t}_{\mathrm{n}}\right): \mathrm{P}\left(\mathrm{t}_{1}, \ldots, \mathrm{t}_{\mathrm{n}}\right) \in \cap \mathrm{M}(\mathrm{LP})\right\} .
$$

$\mathrm{D}(\mathrm{P})$ is the denotation of $\mathrm{P}$ as determined by model-theoretic semantics. Thus modeltheoretic semantics gives a nice characterization of the set of terms computed by a logic program.

On the other hand, algebraic semantics which connects between the theory of tree languages and the semantics of programming languages is now well known and recently introduced to logic programming in [4]. It studies the use of tree languages 
in the semantics of logic programming. In algebraic semantics, the set of terms computed by a logic program LP can be viewed as a tree language. That is to say, the denotation of $P, D(P)=\{t: P(t) \in \cap M(L P)\}$, is a tree language. From the result in [4], a set of trees is rational iff it is computed by a linear monadic logic program, where a rational set of trees is a set of trees which can be recognized by some tree automaton $\mathrm{T}_{\mathrm{A}}$ and a linear monadic logic program is a class of logic programs defined by syntactic restrictions such that predicate symbols are monadic, the height of terms involved is less than or equal to 1 and the variables in a term must be distinct. Therefore, the denotation of $P$ can be written as $D(P)=\{t: t$ is accepted by a tree automaton $\mathrm{T}_{\mathrm{A}}$ about $\mathrm{P}$ in $\left.\mathrm{LP}\right\}$. Based on such an algebraic semantics, we can establish a new inductive inference schema of logic programs so that the problem of inductive inference of logic programs is reduced to the problem of inductive inference of tree automata. By extending an inductive inference algorithm for finite automata [1] to the one for tree automata, we can get an efficient inductive inference method for logic programs. In this setting, the inductive inference algorithm can identify in the limit a class of logic programs, linear monadic logic programs, such that the denotation of $\mathrm{P}$ computed by it is equal to the one in the unknown model.

\section{Basic definitions of trees}

Definition Let $\mathrm{N}$ be the set of positive integers. Dom is a tree domain iff it satisfies

a) Dom $\subseteq N^{*}$ and Dom is finite,

b) Dom is prefix-closed, i.e. if $m, n \in N^{*}$ and $m n \in$ Dom ther $n \in$ Dom,

c) ni $\in$ Dom implies $n j \in$ Dom for $1 \leqq j \leqq i, j \in N$.

A direct successor (direct predecessor) of a node $\mathrm{x}$ is a node $\mathrm{y}$, where $\mathrm{y}=\mathrm{xi}(\mathrm{yi}=\mathrm{x})$ for $i \in N$. The frontier of Dom is the set of all nodes in Dom which have no direct successors. The depth of $\mathrm{n} \in$ Dom is recursively defined as :

$$
\begin{aligned}
& \operatorname{depth}(n)=0 \quad \text { if } n=\varepsilon \\
& \operatorname{depth}(n i)=\operatorname{depth}(n)+1 \quad \text { for } i \in N .
\end{aligned}
$$

If $t$ is a tree domain, then $\operatorname{depth}(t)=\max \{\operatorname{depth}(i): i \in t\}$. 
Definition A ranked alphabet is a pair $(\Gamma, \rho)$ consisting of a finite set $\Gamma$ and a mapping $\rho: \Gamma \rightarrow \mathrm{N}$ which defines the rank of any symbol $f$ in $\Gamma$. For such a set $\Gamma$, we denote by $\Gamma_{n}$ the set $\{f \in \Gamma: \rho(f)=n\}$ for $n \geqq 0$. A tree over a finite ranked alphabet $\Gamma$ is a mapping $t: \operatorname{Dom} \rightarrow \Gamma$, which labels the nodes of the tree domain Dom. We require the following condition which concerns the rank function : if $t(m)=f$ of arity $n \geqq 0$, then for $i \in N, \operatorname{mi} \in \operatorname{Dom}(t)$ iff $1 \leqq i \leqq n$. Let the set of all trees over $\Gamma$ be denoted by $\Gamma^{T}$. If $t \in \Gamma^{T}$, then the subtree of $t$ at $n$, where $n$ is in the domain of $t(n \in \operatorname{Dom}(t))$, is defined as $\mathrm{t} / \mathrm{n}=\{(\mathrm{i}, \mathrm{x}):(\mathrm{ni}, \mathrm{x}) \in \mathrm{t}\}$. For $\mathrm{t} \in \Gamma^{\mathrm{T}}$ and $\mathrm{n} \in \operatorname{Dom}(\mathrm{t})$, the replacement at $\mathrm{n}$ with a tree $\mathrm{u}$ is defined as $t(n \leftarrow u)=\{(m, x): t(m)=x$ and $n \nless m\} \cup\{(n i, x): u(i)=x$ and $i \in \operatorname{Dom}(u)\}$. The replacement (substitution) of terminal nodes labeled $c \in \Gamma$ with a tree $u$ is defined as $t(c \leftarrow u)=\{(m, x): t(m)=x$ and $x \neq c\} \cup\{(n i, x): t(n)=c, u(i)=x$ and $i \in \operatorname{Dom}(u)\}$. Let $\$$ be a new symbol of arity 0 that we add to $\Gamma .(\Gamma \cup\{\$\})^{\mathrm{T}}$ denotes the set of all trees over $\Gamma \cup\{\$\}$. Especially we are interested in the subset Sub of $(\Gamma \cup\{\$\})^{\mathrm{T}}$ which is the set of all trees $t \in(\Gamma \cup\{\$\})^{\mathrm{T}}$ such that $\mathrm{t}$ exactly contains one $\$$-symbol. We use the notation $\Gamma_{\$}{ }^{T}$ for the Sub. For trees $t \in \Gamma^{T}$ and $s \in \Gamma_{\$}^{T}$, we define an operation "." to replace the node labeled $\$$ of $s$ with $t$ by $s \cdot t=s(\$ \leftarrow t)$ (like concatenation of strings).

\section{Tree automaton and linear monadic logic program}

Definition A deterministic (frontier to root) tree automaton over $\Gamma$ is a 4-tuple $\mathrm{T}_{\mathrm{A}}=(\mathrm{Q}, \Gamma, \delta, \mathrm{F})$, where

a) $Q$ is a nonempty finite set of states,

b) $\Gamma$ is a nonempty finite ranked alphabet,

c) $\delta=\left(\delta_{0}, \delta_{1}, \ldots, \delta_{m}\right)$ is a state transition function such that

$$
\delta_{k}: \Gamma_{k} \times Q^{k} \rightarrow Q \quad(k=0,1, \ldots, m),
$$

d) $F \subseteq Q$ is the set of final states.

If $\delta$ is a state transition function from $\Gamma_{k} \times Q^{k}$ to $2^{Q}$, then $T_{A}$ is nondeterministic. $\delta$ can be extended to $\Gamma^{\mathrm{T}}$ by letting :

$$
\begin{aligned}
\delta\left(f\left(t_{1}, \ldots, t_{k}\right)\right) & =\delta_{k}\left(f, \delta\left(t_{1}\right), \ldots, \delta\left(t_{k}\right)\right) \quad \text { for } k>0 \text { and } f \in \Gamma_{k}, \\
& =\delta_{0}(f) \text { for } k=0 \text { and } f \in \Gamma_{0} .
\end{aligned}
$$


The tree $t$ is accepted by $T_{A}$ iff $\delta(t) \in F$. The set of trees accepted by $T_{\Lambda}$ is the subset $\mathrm{L}\left(\mathrm{T}_{\mathrm{A}}\right)$ of $\Gamma^{\mathrm{T}}$ defined as : $\mathrm{L}\left(\mathrm{T}_{\mathrm{A}}\right)=\{\mathrm{t}: \delta(\mathrm{t}) \in \mathrm{F}\}$. A subset $\mathrm{L}$ of $\Gamma^{\mathrm{T}}$ is called rational iff there exists some tree automaton $\mathrm{T}_{\mathrm{A}}$ such that $\mathrm{L}=\mathrm{L}\left(\mathrm{T}_{\mathrm{A}}\right)$.

Example 1 Let $\Gamma=\{t, u, \neg, \bigvee\}, \rho(t)=\rho(u)=0, \rho(\neg)=1, \rho(\bigvee)=2$.

Let $T_{A}=(Q, \Gamma, \delta, F)$ be a tree automaton, where $Q=\{A, B, C\}, F=\{A\}$, $\delta_{0}(\mathrm{t})=\delta_{1}(\neg, \mathrm{B})=\delta_{2}(\bigvee, \mathrm{A}, \mathrm{A})=\delta_{2}(\bigvee, \mathrm{A}, \mathrm{B})=\delta_{2}(\bigvee, \mathrm{A}, \mathrm{C})=\delta_{2}(\bigvee, \mathrm{B}, \mathrm{A})=\delta_{2}(\bigvee, \mathrm{C}, \mathrm{A})=\mathrm{A}$, $\delta_{1}(\neg, \mathrm{A})=\delta_{2}(\bigvee, \mathrm{B}, \mathrm{B})=\mathrm{B}$, $\delta_{0}(\mathrm{u})=\delta_{1}(\neg, \mathrm{C})=\delta_{2}(\bigvee, \mathrm{B}, \mathrm{C})=\delta_{2}(\bigvee, \mathrm{C}, \mathrm{B})=\delta_{2}(\bigvee, \mathrm{C}, \mathrm{C})=\mathrm{C}$.

Then $\mathrm{L}\left(\mathrm{T}_{\mathrm{A}}\right)$ is the set of all true logical formulae over $\Gamma$ interpreted by the Kleene's strong 3-valued connectives.

Note We reserve the predicate symbol $\mathrm{P}$ for the inferring predicate.

Let the least Herbrand model for a logic program LP be denoted by $\cap M(L P)$.

Definition ([4]) A linear monadic logic program is a logic program in which all predicate symbols are monadic and all the terms occurring in atomic formulas are of one of the following two forms :

a) $x_{i} \quad(i \in N)$

b) $\mathrm{f}\left(\mathrm{x}_{\mathrm{i}_{1}}, \ldots, \mathrm{x}_{\mathrm{i}_{\mathrm{m}}}\right)$ with $\mathrm{f} \in \Gamma_{\mathrm{m}},\left\{\mathrm{i}_{1}, \ldots, \mathrm{i}_{\mathrm{m}}\right\} \subseteq \mathrm{N}$ the $\mathrm{i}_{\mathrm{k}}$ being pairwise distinct.

Now we state very important theorem from [4] which connects a linear monadic logic program with a tree automaton.

Proposition 3.1 ([4]) A set of trees is rational iff it can be computed by a linear monadic logic program.

By the results of logic programs in [2], we can restate the above theorem as follows.

Corollary 3.2 If LMLP is a linear monadic logic program and $\mathrm{P}$ is a predicate symbol in LMLP, then the set of trees $\{\mathrm{t}: \mathrm{P}(\mathrm{t}) \in \cap M(L M L P)\}$ is rational. Conversely, if a set of 
trees $\mathrm{T}$ is rational, then there is a linear monadic logic program LMLP such that $\mathrm{T}=\{\mathrm{t}: \mathrm{P}(\mathrm{t}) \in \cap \mathrm{M}(\mathrm{LMLP})\}$ for some predicate symbol $\mathrm{P}$ in LMLP.

Definition-A Let $T_{A}=(Q, \Gamma, \delta, F)$ be a tree automaton. We define a set of predicate symbols $R=\left\{R_{q}: q \in Q\right\}$ in one-to-one correspondence with the set of states of the $T_{A}$. To code the computation of $T_{A}$, we need a clause for each transition. So, for each $f \in \Gamma n$ and each $n$-tuple of states $\left(q_{1}, \ldots, q_{n}\right)$, we define the clause $C_{f, q_{1}, \ldots, q_{n}}$ as :

$$
\mathrm{C}_{f, \mathrm{q}_{1}, \ldots, \mathrm{q}_{\mathrm{n}}}=\mathrm{R}_{\delta\left(f, \mathrm{q}_{1}, \ldots, \mathrm{q}_{\mathrm{n}}\right)}\left(\mathrm{f}\left(\mathrm{x}_{1}, \ldots, \mathrm{x}_{\mathrm{n}}\right)\right) \leftarrow \mathrm{R}_{\mathrm{q}_{1}}\left(\mathrm{x}_{1}\right), \ldots, \mathrm{R}_{\mathrm{q}_{\mathrm{n}}}\left(\mathrm{x}_{\mathrm{n}}\right) .
$$

Another set of clauses is necessary to take care of the set of final states. So, for each $q \in F$, we define the clause $C_{q}^{\prime}$ as :

$$
C^{\prime}{ }_{q}=P(x) \leftarrow R_{q}(x) \text {. }
$$

Example 2 Let $\mathrm{T}_{\mathrm{A}}=(\mathrm{Q}, \Gamma, \delta, \mathrm{F})$ be a tree automaton as in example 1 . 'The corresponding linear monadic logic program is the following set of clauses.

$$
\begin{aligned}
& P(x) \leftarrow R_{A}(x) . \\
& R_{A}(t) \leftarrow . \\
& R_{C}(u) \leftarrow . \\
& R_{A}(\neg x) \leftarrow R_{B}(x), \\
& R_{B}(\neg x) \leftarrow R_{A}(x) \\
& R_{C}(\neg x) \leftarrow R_{C}(x) . \\
& R_{A}(x \vee y) \leftarrow R_{A}(x), R_{A}(y) . \\
& R_{A}(x \vee y) \leftarrow R_{A}(x), R_{B}(y) . \\
& R_{A}(x \vee y) \leftarrow R_{A}(x), R_{C}(y) . \\
& R_{A}(x \vee y) \leftarrow R_{B}(x), R_{A}(y) \\
& R_{A}(x \vee y) \leftarrow R_{C}(x), R_{A}(y) . \\
& R_{B}(x \vee y) \leftarrow R_{B}(x), R_{B}(y) . \\
& R_{C}(x \vee y) \leftarrow R_{B}(x), R_{C}(y) . \\
& R_{C}(x \vee y) \leftarrow R_{C}(x), R_{B}(y) . \\
& R_{C}(x \vee y) \leftarrow R_{C}(x), R_{C}(y) .
\end{aligned}
$$

This is a logic program for determining the truth of a logical formula.

Proposition 3.3 Let $\mathrm{T}_{\mathrm{A}}=(\mathrm{Q}, \mathrm{\Gamma}, \delta, \mathrm{F})$ be a tree automaton and LMLP be the corresponding linear monadic logic program in the sense of Definition-A. Then $\mathrm{R}_{\mathrm{q}}(\mathrm{t}) \in \cap \mathrm{M}(\mathrm{LMLP})$ iff $\delta(\mathrm{t})=\mathrm{q}$. Furthermore, $\mathrm{P}(\mathrm{t}) \in \cap \mathrm{M}(\mathrm{LMLP})$ iff $\delta(\mathrm{t})$ is in $\mathrm{F}$. 
(Proof) We prove it by induction on the depth of $t$. Suppose first that the depth of $t$ is 0 , i.e. $t=a \in \Gamma_{0}$. By the definition of $C_{f, q_{1}, \ldots, q_{n}}$, there is a clause $R_{\delta(a)}(a) \leftarrow$ in LMLP. Then clearly $R_{\delta(a)}(a) \in \cap M(L M L P)$. If $\delta(a)=q$, then $R_{q}(a)=R_{\delta(a)}(a) \in \cap M(L M L P)$. Conversely if $R_{q}(a) \in \cap M(L M L P)$, since $T_{\Lambda}$ is deterministic (so $\delta$ is deterministic), $\delta(a)=q$.

Next suppose that the result holds for all trees with depth at most $h$. Let $t$ be a tree of depth $h+1$, so that $t=f\left(u_{1}, \ldots, u_{n}\right)$ for some trees $u_{1}, \ldots, u_{n}$ with depth at most $h$ and some $f \in \Gamma_{n}$. For the if part, assume that $\delta(t)=q$. By the definition of $\delta$, $\delta(t)=\delta\left(f\left(u_{1}, \ldots, u_{n}\right)\right)=\delta\left(f, \delta\left(u_{1}\right), \ldots, \delta\left(u_{n}\right)\right)=q$. By the definition of the clause $C_{f, q_{1}, \ldots, q_{n}}$, there is a clause $R_{\delta\left(f, \delta\left(u_{1}\right), \ldots, \delta\left(u_{n}\right)\right)}\left(f\left(x_{1}, \ldots, x_{n}\right)\right) \leftarrow R_{\delta\left(u_{1}\right)}\left(x_{1}\right), \ldots, R_{\delta\left(u_{n}\right)}\left(x_{n}\right)$ in LMLP. For $1 \leqq \mathrm{i} \leqq \mathrm{n}$, by the induction hypothesis, $\mathrm{R}_{\delta\left(\mathrm{u}_{\mathrm{i}}\right)}\left(\mathrm{u}_{\mathrm{i}}\right) \in \cap \mathrm{M}(\mathrm{LMLP})$ iff $\delta\left(\mathrm{u}_{\mathrm{i}}\right)=\delta\left(\mathrm{u}_{\mathrm{i}}\right)$. The righthand side of this statement is obviously true. Thus $\left.R_{\delta\left(u_{i}\right.}\right)\left(u_{i}\right) \in \cap M(L M L P)$, and so $R_{\delta\left(f, \delta\left(u_{1}\right), \ldots, \delta\left(u_{n}\right)\right)}\left(f\left(u_{1}, \ldots, u_{n}\right)\right) \in \cap M(L M L P)$. Then

$$
\begin{aligned}
R_{\mathrm{q}}(\mathrm{t})= & \mathrm{R}_{\delta(\mathrm{t})}(\mathrm{t}), \text { by the assumption, } \\
& =\mathrm{R}_{\delta\left(\mathrm{f}\left(\mathrm{u}_{1}, \ldots, \mathrm{u}_{\mathrm{n}}\right)\right)}\left(\mathrm{f}\left(\mathrm{u}_{1}, \ldots, \mathrm{u}_{\mathrm{n}}\right)\right) \\
& =\mathrm{R}_{\delta\left(f, \delta\left(\mathrm{u}_{1}\right), \ldots, \delta\left(\mathrm{u}_{\mathrm{n}}\right)\right)\left(\mathrm{f}\left(\mathrm{u}_{1}, \ldots, \mathrm{u}_{\mathrm{n}}\right)\right), \text { by the definition of } \delta .}
\end{aligned}
$$

Hence $R_{q}(t) \in \cap M(L M L P)$.

For the only-if part, assume that $R_{q}(t) \in \cap M(L M L P)$. Then $R_{q}\left(f\left(u_{1}, \ldots, u_{n}\right)\right) \in \cap M(L M L P)$. For $R_{q}\left(f\left(u_{1}, \ldots, u_{n}\right)\right)$, there is a ground instance $R_{\delta\left(f, q_{1}, \ldots, q_{n}\right)}\left(f\left(u_{1}, \ldots, u_{n}\right)\right) \leftarrow R_{q_{1}}\left(u_{1}\right), \ldots, R_{q_{n}}\left(u_{n}\right)$ of a clause in LMLP such that $\delta\left(f, q_{1}, \ldots, q_{n}\right)=q$ and $R_{q_{1}}\left(u_{1}\right), \ldots, R_{q_{n}}\left(u_{n}\right) \in \cap M(L M L P)$. By the induction hypothesis, $\delta\left(u_{i}\right)=q_{i}(1 \leqq i \leqq n)$. Then

$$
\begin{aligned}
\delta(t) & =\delta\left(f\left(u_{1}, \ldots, u_{n}\right)\right) \\
& =\delta\left(f, \delta\left(u_{1}\right), \ldots, \delta\left(u_{n}\right)\right), \quad \text { by the definition of } \delta, \\
& =\delta\left(f, q_{1}, \ldots, q_{n}\right) \\
& =q .
\end{aligned}
$$

This completes the induction.

Furthermore, if $\delta(t)$ is in $F$, there is a final state $q_{f}$ in $F$ such that $\delta(t)=q_{f}$. Then by the above result, $R_{q_{f}}(t) \in \cap M(L M L P)$, and by the definition of $C_{q}^{\prime}, P(t) \in \cap M(L M L P)$. 
Conversely if $P(t) \in M(L M L P)$, there is a ground instance $P(t) \leftarrow R_{q}(t)$ of a clause in LMLP such that $R_{q}(t) \in \cap M(L M L P)$ and $q$ is a final state. By the above result, $\delta(t)=q$, and hence $\delta(t)$ is in F. Q.E.D.

By the above result, in the inductive inference schema of linear monadic logic program, we have only to consider inferring a linear monadic logic program of the form in Definition-A.

\section{Predicate characterization matrix}

Definition A set of test predicates $\mathrm{S}$ is a finite set of trees of $\Gamma^{\mathrm{T}}$. The set of test clauses is defined to be $X(S)=\left\{f(\bar{u}): f \in \Gamma_{i}, \bar{u} \in S^{i}\right.$, and $f(\bar{u}) \notin S$ for $\left.i \geqq 1\right\}$. A set of experiments $E$ is a finite set of trees of $\Gamma_{\$}{ }^{T}$. $S$ is called subtree-closed if $s \in S$ implies all subtrees of $s$ are elements of S. E is called \$-prefix-closed with respect to $\mathrm{S}$ if $\mathrm{e} \in \mathrm{E}$ except $\$$ implies there exists an $e^{\prime}$ in $E$ such that $e=e^{\prime} \cdot f\left(s_{1}, \ldots, s_{i-1}, \$, s_{i}, \ldots, s_{n-1}\right)$ for some $f \in \Gamma_{n}$, $s_{1}, \ldots, s_{n-1} \in S$ and $i(1 \leqq i \leqq n)$.

Definition A predicate characterization matrix is a triple (S, E, M) where $M$ is a matrix with labeled rows and columns such that

1) The rows are labeled with the elements of $S \cup X(S)$.

2) The columns are labeled with the elements of $E$.

3) Each entry of $M$ is either 0 or 1.

4) If $s_{i}, s_{j} \in S \cup X(S)$ and $e_{i}, e_{j} \in E$ and $e_{i} \cdot s_{i}=e_{j} \cdot s_{j}$, then the $\left(s_{i}, e_{i}\right)$ and $\left(s_{j}, e_{j}\right)$ positions in $M$ must have the same entry.

The data contained in $M$ is $D(M)=\{(e \cdot s, y): s \in S \cup X(S), e \in E$, and the entry of $M$ is $y \in\{0,1\}\}$. Thus we can regard $D(M)$ as a finite function mapping $E \cdot(\operatorname{S} \cup X(S))$ to $\{0,1\}$. If $s$ is an element of $(\mathrm{S} \cup \mathrm{X}(\mathrm{S}))$, then $\operatorname{row}(\mathrm{s})$ denotes the finite function $\mathrm{f}$ from $\mathrm{E}$ to $\{0,1\}$ defined by $f(e)=D(M)(e \cdot s)$.

A predicate characterization matrix is called closed if every row $(\mathrm{x})$ of test clause $x \in X(S)$ is identical to some row $(s)$ of test predicate $s \in S$. A predicate characterization matrix is called consistent if whenever $s_{1}$ and $s_{2}$ are test predicates of $S$ such that 
row $\left(s_{1}\right)$ is equal to row $\left(s_{2}\right)$, for all $f \in \Gamma_{n}$ and $u_{1}, \ldots, u_{n}-1 \in S$, $\operatorname{row}\left(f\left(u_{1}, \ldots, u_{i-1}, s_{1}, u_{i}, \ldots, u_{n-1}\right)\right)$ is equal to $\operatorname{row}\left(f\left(u_{1}, \ldots, u_{i-1}, s_{2}, u_{i}, \ldots, u_{n-1}\right)\right)$ for $0 \geqslant i<n$ $(n \geqq 0)$.

The ideas of the closed, consistent predicate characterization matrix and the algorithm using this are essentially the extensions of Angluin's ones [1] (the extension from finite automata to tree automata and so to linear monadic logic programs). A sequence of following lemmas and theorems are guided by those Angluin's results. The idea of the characterization matrix is also related to the work by Gold [3].

Definition Let (S, E, M) be a closed, consistent predicate characterization matrix such that $\mathrm{E}$ contains $\$$. The constructed linear monadic logic program LMLP $\mathrm{M}_{\mathrm{M}}$ over $\Gamma$ from $(S, E, M)$ is defined with predicate set Predicate, calling predicate $P$, and the set of clauses $L_{M L P}$ as follows.

$$
\begin{aligned}
\text { Predicate } & =\left\{R_{\text {row }(s)}(x): s \in S\right\} \\
\operatorname{LMLP}_{M}= & \left\{P(x) \leftarrow R_{\text {row }(s)}(x): s \in S \text { and } D(M)(s)=1\right\} \\
& \cup\left\{R_{\text {row }\left(f\left(s_{1}, \ldots, s_{n}\right)\right)}\left(f\left(x_{1}, \ldots, x_{n}\right)\right) \leftarrow R_{\text {row }\left(s_{1}\right)}\left(x_{1}\right), \ldots, R_{\text {row }\left(s_{n}\right)}\left(x_{n}\right): f \in I_{n}, n>0\right\} \\
& U\left\{R_{\text {row }(a)}(a) \leftarrow: a \in \Gamma_{0}\right\} .
\end{aligned}
$$

Lemma 4.1 Suppose that (S, E, M) is a closed, consistent predicate characterization matrix such that $\mathrm{S}$ is subtree-closed and $\mathrm{E}$ is $\$$-prefix-closed with respect to $\mathrm{S}$. For the constructed linear monadic logic program $\mathrm{LMLP}_{\mathrm{M}}$ and for every $\mathrm{s}$ in (SUX(S)), $R_{\text {row (s) }}(s) \in \cap M(L M L P M)$.

(Proof) We prove it by induction on the depth of $\mathrm{s}$. Suppose first that the depth of $\mathrm{s}$ is 0 , i.e., $s \in \Gamma_{0}$. Since $R_{\text {row }}(s)(s) \leftarrow$ by the definition of $L M L P_{M}$, the result is clearly true. Next suppose that the result holds for all trees in (SUX(S)) with depth at most $h$. Let $t$ in $(S \cup X(S))$ have depth $h+1$, so that $t=f\left(s_{1}, \ldots, s_{n}\right)$ for some trees $s_{1}, \ldots, s_{n}$ over $\Gamma$ with depth at most $h$ and some $f$ in $\Gamma_{n}$. Since $S$ is subtree-closed, $s_{1}, \ldots, s_{n}$ must be in $S$. Then 
$R_{\text {row }(t)}(t) \in \cap M\left(L M L P_{M}\right)$

iff $R_{\text {row }\left(f\left(s_{1}, \ldots, s_{n}\right)\right)}\left(f\left(s_{1}, \ldots, s_{n}\right)\right) \in \cap M\left(L M L P_{M}\right)$

iff $R_{\text {row }\left(s_{1}\right)}\left(s_{1}\right), \ldots, R_{\text {row }\left(s_{n}\right)}\left(s_{n}\right) \in \cap M\left(L M L P_{M}\right)$,

by the definition of $\mathrm{LMLP}_{M}$.

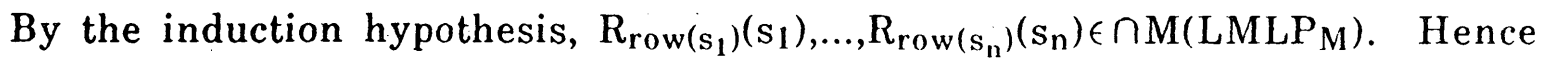
$R_{\text {row }(t)}(t) \in \cap M(L M L P M)$ is true. Q.E.D.

Lemma 4.2 Suppose that (S, E, M) is a closed, consistent predicate characterization matrix such that $\mathrm{S}$ is subtree-closed and $\mathrm{E}$ is $\$$-prefix-closed with respect to $\mathrm{S}$. For the constructed linear monadic logic program $\mathrm{LMLP}_{\mathrm{M}}$ and for any tree $\mathrm{t}$ over $\mathrm{\Gamma}$, there is exactly one function value row(s) such that $\mathrm{R}_{\text {row }(\mathrm{s})}(\mathrm{t}) \in \cap \mathrm{M}\left(\mathrm{LMLP}_{\mathrm{M}}\right)$ and $\mathrm{s} \in \mathrm{S}$.

(Proof) We prove it by the induction on the depth of $t$. Suppose first that the depth of $t$ is 0 , i.e. $t=a \in \Gamma_{0}$. By the definition of $L M L P_{M}$, for $a \in \Gamma_{0}$, row(a) is exactly one function value such that $R_{\text {row(a) }}(a) \in \cap M\left(L M L P_{M}\right)$ and $a \in S$. Next suppose that the result holds for all trees with depth at most $h$. Let $t$ be a tree of depth $h+1$, so that $t=f\left(u_{1}, \ldots, u_{n}\right)$ for some trees $u_{1}, \ldots, u_{n}$ with depth at most $h$ and some $f$ in $\Gamma_{n}$. There are several clauses of the form : $R_{\text {row }\left(f\left(v_{1}, \ldots v_{n}\right)\right)}\left(f\left(x_{1}, \ldots, x_{n}\right)\right) \leftarrow R_{\text {row }\left(v_{1}\right)}\left(x_{1}\right), \ldots, R_{\text {row }\left(v_{n}\right)}\left(x_{n}\right)$ in LMLP $_{M}$. However by the induction hypothesis, for each $u_{i}(1 \leqq i \leqq n)$, there is exactly one function value, say row $\left(s_{i}\right)$, such that $\left.R_{\text {row }\left(s_{i}\right.}\right)\left(u_{i}\right) \in \cap M(L M L P M)$ and $s_{i} \in S$. Since $(\mathrm{S}, \mathrm{E}, \mathrm{M})$ is consistent, there is only one clause of the form :

$R_{\text {row }\left(f\left(s_{1}, \ldots s_{n}\right)\right)}\left(f\left(x_{1}, \ldots, x_{n}\right)\right) \leftarrow R_{\text {row }\left(s_{1}\right)}\left(x_{1}\right), \ldots, R_{\text {row }\left(s_{n}\right)}\left(x_{n}\right)$ in LMLP $M$.

Thus row $\left(f_{(}\left(s_{1}, \ldots, s_{n}\right)\right)$ is exactly one function value such that $R_{\text {row }\left(f\left(s_{1}, \ldots s_{n}\right)\right)}\left(f\left(u_{1}, \ldots, u_{n}\right)\right) \in \cap M(L M L P M)$, and since $(S, E, M)$ is closed, row $\left(f\left(s_{1}, \ldots, s_{n}\right)\right)$ is equal to row(s) for some $\mathrm{s}$ in $\mathrm{S}$. Hence there is exactly one function value row(s) such that $R_{\text {row }(s)}(t) \in \cap M(L M L P M)$ and $s \in S$. Q.E.D.

Lemma 4.3 (replacement) Suppose that (S, E, M) is a closed, consistent predicate characterization matrix such that $\mathrm{S}$ is subtree-closed and $\mathrm{E}$ is \$-prefix-closed with respect to $\mathrm{S}$ and that $\mathrm{LMLP}_{\mathrm{M}}$ is the constructed linear monadic logic program. Suppose that $\mathrm{R}_{\text {row }(\mathrm{s})}(\mathrm{t}) \in \cap M\left(\mathrm{LMLP}_{\mathrm{M}}\right), \mathrm{R}_{\text {row }}\left(\mathrm{s}^{\prime}\right)\left(\mathrm{t}^{\prime}\right) \in \cap M\left(L M L P_{M}\right)$ and $\operatorname{row}(\mathrm{s})=\operatorname{row}\left(\mathrm{s}^{\prime}\right)$ 
for $\mathrm{s}$, $\mathrm{s}^{\prime}$ in $(\mathrm{SUX}(\mathrm{S}))$ and trees $\mathrm{t}, \mathrm{t}^{\prime}$ over $\Gamma$. For e in $\mathrm{E}, \mathrm{P}(\mathrm{e} \cdot \mathrm{t}) \in \mathrm{\cap M}\left(\mathrm{LMLP}_{\mathrm{M}}\right)$ iff $\mathrm{P}\left(\mathrm{e} \cdot \mathrm{t}^{\prime}\right) \in \cap M\left(L M L P_{M}\right)$.

(Proof) We prove it by induction on the depth of $\$$ in e. When e is $\$$, if $P(e \cdot t)=P(t) \in \cap M\left(L M L P_{M}\right)$, then there is a ground instance $\left.P(t) \leftarrow R_{\text {row }\left(s_{0}\right)}\right)(t)$ of a clause $P(x) \leftarrow R_{\text {row }\left(s_{0}\right)}(x)$ in $L M L P_{M}$ such that $R_{\text {row }\left(s_{0}\right)}(t) \in \cap M\left(L M L P_{M}\right)$ and $s_{0} \in S$. By lemma 4.2, row $\left(\mathrm{s}_{0}\right)=$ row $(\mathrm{s})$. By the assumption, row $\left(\mathrm{s}_{0}\right)=$ row $\left(\mathrm{s}^{\prime}\right)$ and $\left.R_{\text {row }\left(s^{\prime}\right)\left(t^{\prime}\right) \in \cap M(L M L P}^{M}\right)$. Hence $P\left(t^{\prime}\right) \in \cap M(L M L P M)$. Interchanging the roles of $s$ and $s^{\prime}$ and of $t$ and $t$ ', we obtain the converse.

Next suppose that the result holds for all e in $\mathrm{E}$ where the depth of $\$$ is at most $\mathrm{h}$. Let e be an element of $E$ where the depth of $\$$ is $h+1$. Since $E$ is $\$$-prefix-closed with respect to $\mathrm{S}, \mathrm{e}=\mathrm{e}^{\prime} \cdot \mathrm{f}\left(\mathrm{s}_{1}, \ldots, \mathrm{Si}_{\mathrm{i}-1}, \$, \mathrm{Si}_{1}, \ldots, \mathrm{S}_{\mathrm{n}-1}\right)$ for some $\mathrm{f} \in \Gamma_{\mathrm{n}}, \mathrm{s}_{1}, \ldots, \mathrm{S}_{\mathrm{n}-1} \in \mathrm{S}, \mathrm{i}(1 \leqq i \leqq n)$ and some $e^{\prime}$ in $E$ where the depth of $\$$ is $h$. Since (S, E, M) is closed, there is some so in $\mathrm{S}$ such that $\operatorname{row}\left(\mathrm{s}_{0}\right)=\operatorname{row}(\mathrm{s})$. Then $R_{\text {row }\left(s_{0}\right)}(t) \in \cap M\left(L M L P_{M}\right)$ and by lemma 4.1, $R_{\text {row }\left(s_{1}\right)}\left(s_{1}\right), \ldots, R_{\text {row }\left(s_{n-1}\right)}\left(s_{n-1}\right) \in \cap M\left(L M L P_{M}\right)$. By the definition of LMLP $P_{M}$, there is a clause of the form

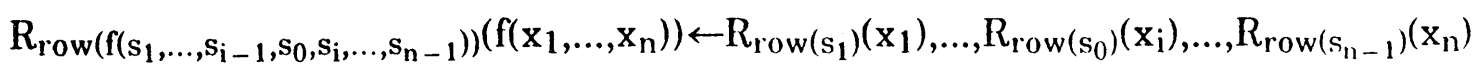

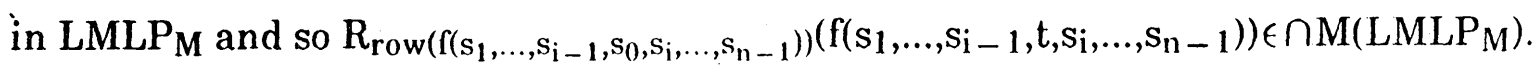
Since $\operatorname{row}\left(\mathrm{s}_{0}\right)=\operatorname{row}\left(\mathrm{s}^{\prime}\right)$ and $\mathrm{R}_{\text {row }}\left(\mathrm{s}^{\prime}\right)\left(\mathrm{t}^{\prime}\right) \in \cap M\left(L M L P_{M}\right)$, $\left.R_{\text {row }\left(f\left(s_{1}, \ldots, s_{i-1}, s_{0}, s_{i}, \ldots, s_{n-1}\right)\right)\left(f\left(s_{1}, \ldots, S_{i-1}, t^{\prime}, s_{i}, \ldots, s_{n-1}\right)\right) \in \cap M(L M L P}^{M}\right)$.

By the induction hypothesis, $P\left(e^{\prime} \cdot f\left(s_{1}, \ldots, s_{-1}, t, s_{i}, \ldots, s_{n-1}\right)\right) \in \cap M(L M L P M)$ iff $\mathrm{P}\left(\mathrm{e}^{\prime} \cdot \mathrm{f}\left(\mathrm{s}_{1}, \ldots, \mathrm{s}_{\mathrm{i}-1}, \mathrm{t}^{\prime}, \mathrm{s}_{\mathrm{i}}, \ldots, \mathrm{s}_{\mathrm{n}-1}\right)\right) \in \cap M\left(L_{M L P}\right)$. Therefore $\mathrm{P}(\mathrm{e} \cdot \mathrm{t}) \in \cap M(L M L P M)$ iff $\mathrm{P}\left(\mathrm{e} \cdot \mathrm{t}^{\prime}\right) \in \cap M\left(L_{M L P}\right)$, Q.E.D.

Theorem 4.4 Suppose that (S, E, M) is a closed, consistent predicate characterization matrix such that $\mathrm{S}$ is subtree-closed and $\mathrm{E}$ is \$-prefix-closed with respect to $\mathrm{S}$. Then the constructed linear monadic logic program $\mathrm{LMLP}_{\mathrm{M}}$ agrees with the data in $\mathrm{M}$. That is, for every tree $\mathrm{s}$ in $(\mathrm{SUX}(\mathrm{S}))$ and $\mathrm{e}$ in $\mathrm{E}, \mathrm{P}(\mathrm{e} \cdot \mathrm{s}) \in \mathrm{MM}\left(\mathrm{LMLP}_{\mathrm{M}}\right)$ iff $\mathrm{D}(\mathrm{M})(\mathrm{e} \cdot \mathrm{s})=1$. (Proof) We prove it by induction on the depth of $\$$ in e. When e is $\$$ and $s$ is any element of $(\operatorname{SUX}(S))$, by lemma 4.1, $R_{\text {row }(e \cdot s)}(e \cdot s)=R_{\text {row }(s)}(s) \in \cap M(L M L P M)$. If $s$ is in 
$S$, then by the definition of $\operatorname{LMLP}_{M}, P(x) \leftarrow R_{\text {row }(s)}(x)$ in $\operatorname{LMLP}_{M}$ iff $D(M)(s)=1$. Hence $P(s) \in \cap M(L M L P M)$ iff $D(M)(s)=1$. If $s$ is in $X(S)$, then since $(S, E, M)$ is closed, $\operatorname{row}\left(s^{\prime}\right)=\operatorname{row}\left(s^{\prime}\right)$ for some $s^{\prime}$ in $S$, and $P(x) \leftarrow R_{\text {row }\left(s^{\prime}\right)}(x)$ in $L M L P M$ iff $D(M)\left(s^{\prime}\right)=1$, and so $P(x) \leftarrow R_{\text {row(s) }}(x)$ in $L_{M L P}$ iff $D(M)(s)=1$. Hence $P(s) \in \cap M(L M L P M)$ iff $\mathrm{D}(\mathrm{M})(\mathrm{s})=1$.

Next suppose that the result holds for all e in $E$ where the depth of $\$$ is at most $h$. Let e be an element of $E$ where the depth of $\$$ is $h+1$. Since $E$ is $\$$-prefix-closed with respect to $S, e=e^{\prime} \cdot f\left(s_{1}, \ldots, s_{i-1}, \$, s_{i}, \ldots, s_{n-1}\right)$ for some $f \in \Gamma_{n}, s_{1}, \ldots, s_{n-1} \in S, i(1 \leqq i=n)$ and some $e^{\prime}$ in $E$ where the depth of $\$$ is $h$. For any element s of (SUX(S)), since (S, E, M) is closed, there is an element $\mathrm{s}^{\prime}$ in $\mathrm{S}$ such that row $(\mathrm{s})=$ row $\left(\mathrm{s}^{\prime}\right)$. By lemma 4.1, $R_{\text {row }(s)}(s) \in \cap M\left(L M L P_{M}\right)$ and $R_{\text {row }\left(s^{\prime}\right)}\left(s^{\prime}\right) \in \cap M\left(L M L P_{M}\right)$. Then by replacement lemma 4.3 ,

$$
\begin{aligned}
& P(e \cdot s) \in \cap M\left(L M L P_{M}\right) \\
& \text { iff } P\left(e \cdot s^{\prime}\right) \in \cap M\left(L M L P_{M}\right) \\
& \text { iff } P\left(e^{\prime} \cdot f\left(s_{1}, \ldots, s_{i-1}, \$, s_{i}, \ldots, s_{n-1}\right) \cdot s^{\prime}\right) \in \cap M\left(L M L P_{M}\right) \\
& \text { iff } P\left(e^{\prime} \cdot f\left(s_{1}, \ldots, s_{i-1}, s^{\prime}, s_{i}, \ldots, s_{n-1}\right)\right) \in \cap M\left(L M L P_{M}\right)
\end{aligned}
$$

By the induction hypothesis,

$P\left(e^{\prime} \cdot f\left(s_{1}, \ldots, s_{i-1}, s^{\prime}, s_{i}, \ldots, s_{n-1}\right)\right) \in \cap M\left(L M L P_{M}\right)$ iff $D(M)\left(e^{\prime} \cdot f\left(s_{1}, \ldots, s_{i-1}, s^{\prime}, s_{i}, \ldots, s_{n-1}\right)\right)=1$.

Since $\operatorname{row}(\mathrm{s})=\operatorname{row}\left(\mathrm{s}^{\prime}\right)$ and $(\mathrm{S}, \mathrm{E}, \mathrm{M})$ is consistent,

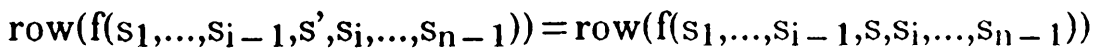
and hence $D(M)\left(e^{\prime} \cdot f\left(s_{1}, \ldots, s_{i}-1, s^{\prime}, s_{i}, \ldots, s_{n}-1\right)\right)=D(M)\left(e^{\prime} \cdot f\left(s_{1}, \ldots, s_{i}-1, s_{,} s_{i}, \ldots, s_{n}-1\right)\right)$, and since $e^{\prime} \cdot f\left(s_{1}, \ldots, s_{i-1}, \$, s_{i}, \ldots, s_{n-1}\right)=e$ is in $E, D(M)\left(e^{\prime} \cdot f\left(s_{1}, \ldots, s_{i-1}, s_{,} s_{i}, \ldots, s_{n-1}\right)\right)$ $=D(M)(e \cdot s)$. 'Therefore $P(e \cdot s) \in \cap M(L M L P M)$ iff $D(M)(e \cdot s)=1$. Q.E.D.

For the proof of the next result, for a tree automaton $T_{\Lambda}=(Q, \Gamma, \delta, F)$ we extend $\delta$ to $(\Gamma \cup Q)^{\mathrm{T}}$ by letting : $\delta(q)=q$ for $q \in Q$, where $Q$ is considered as a set of 0 -ary constant symbols. In this definition, if $q=\delta(s)$ for $q \in Q$ and $s \in \Gamma^{T}$, then $\delta(t(x \leftarrow q))=\delta(t(x \leftarrow s))$ for $t \in \Gamma^{\mathrm{T}}$ and $\mathrm{x} \in \operatorname{Dom}(\mathrm{t})$ 
'Theorem 4.5 Suppose that (S, E, M) is a closed, consistent predicate characterization matrix such that $\mathrm{S}$ is subtree-closed and $\mathrm{E}$ is $\$$-prefix-closed with respect to $\mathrm{S}$. Suppose that the constructed linear monadic logic program $\mathrm{LMLP}_{\mathrm{M}}$ from $(\mathrm{S}, \mathrm{E}, \mathrm{M})$ has $\mathrm{n}$ predicates. If $\mathrm{T}_{\mathrm{A}}=(\mathrm{Q}, \Gamma, \delta, \mathrm{F})$ is any tree automaton which agrees with the data in $\mathrm{M}$ that has $\mathrm{n}$ or fewer states and $\mathrm{LMLP}^{\prime} \mathrm{I}_{\Lambda}$ is a corresponding linear monadic logic program in the sense of Definition-A, then $\mathrm{LMLP}_{\mathrm{M}}$ is isomorphic to $\mathrm{LMLP}_{\mathrm{T}} \mathrm{P}_{\Lambda}$.

(Proof) We prove it by exhibiting an isomorphism $\phi$. First define, for each $q$ in $Q$, $\operatorname{row}(q)$ to be the finite function $f$ from $E$ to $\{0,1\}$ such that $f(e)=1$ iff $\delta(e \cdot q)$ is in $F$. Since $T_{A}$ agrees with the data in $M$, for each $s$ in $(S U X(S))$ and each e in $E, \delta(e \cdot s)$ is in $\mathrm{F}$ iff $\mathrm{D}(\mathrm{M})(\mathrm{e} \cdot \mathrm{s})=1$, so row $(\delta(\mathrm{s}))$ is equal to row $(\mathrm{s})$ in $(\mathrm{S}, \mathrm{E}, \mathrm{M})$. Hence as s ranges over all of $S$, row $(\delta(s))$ ranges over all the elements of $Q$, so $T_{\Lambda}$ must have at least $n$ states, i.e., it must have exactly $n$ states. Thus, for each $\mathrm{s}$ in $\mathrm{S}$ there is a unique $q$ in $\mathrm{Q}$ such that $\operatorname{row}(\mathrm{s})=\operatorname{row}(\mathrm{q})$, namely, $\delta(\mathrm{s})$. Next define for each $\mathrm{s}$ in $\mathrm{S}, \phi(\operatorname{row}(\mathrm{s}))$ to be $\delta(\mathrm{s})$. This mapping is one-to-one and onto. Then extend $\phi$ to define for each predicate in $\operatorname{LMLP}_{M}, \phi\left(\mathrm{R}_{\text {row(s) }}\right)$ to be $\mathrm{R}_{\phi(\text { row(s)). }}$. We must verify that it preserves the clauses. For each $s_{1}, \ldots, s_{n}$ in $S$ and $f \in \Gamma_{n}$, let $s$ be an element of $S$ such that $\operatorname{row}\left(f\left(s_{1}, \ldots, s_{n}\right)\right)=\operatorname{row}(s)$. Then

$$
\begin{aligned}
\left.\phi\left(R_{\operatorname{row}}\left(\mathrm{f}_{(}, \ldots, \mathrm{s}_{\mathrm{n}}\right)\right)(\mathrm{x})\right) & =\mathrm{R}_{\phi\left(\operatorname{row}\left(\mathrm{f}\left(\mathrm{s}_{1}, \ldots, \mathrm{s}_{\mathrm{n}}\right)\right)\right)(\mathrm{x})} \\
& =\mathrm{R}_{\phi(\operatorname{row}(\mathrm{s}))(\mathrm{x})} \\
& =\mathrm{R}_{\delta(\mathrm{s})}(\mathrm{x})
\end{aligned}
$$

Also,

$$
R_{\delta\left(f, \delta\left(s_{1}\right), \ldots, \delta\left(s_{n}\right)\right)}(x)=R_{\delta\left(f\left(s_{1}, \ldots, s_{n}\right)\right)}(x)
$$

Since $\delta(s)$ and $\delta\left(f\left(s_{1}, \ldots, s_{n}\right)\right)$ have identical row values, namely row $(s)$ and

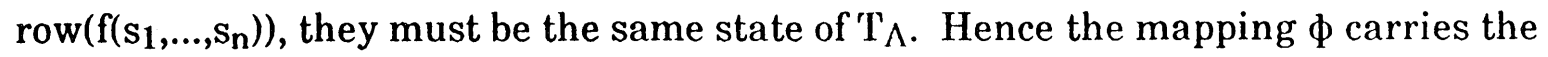

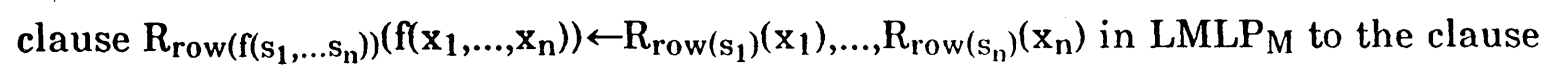
$\mathrm{R}_{\delta\left(f, \delta\left(\mathrm{s}_{1}\right), \ldots, \delta\left(\mathrm{s}_{\mathrm{n}}\right)\right)}\left(\mathrm{f}\left(\mathrm{x}_{1}, \ldots, \mathrm{x}_{\mathrm{n}}\right)\right) \leftarrow \mathrm{R}_{\delta\left(\mathrm{s}_{1}\right)}\left(\mathrm{x}_{1}\right), \ldots, \mathrm{R}_{\delta\left(\mathrm{s}_{\mathrm{n}}\right)}\left(\mathrm{x}_{\mathrm{n}}\right)$ in $\mathrm{LMLP} \mathrm{T}_{\mathrm{T}^{\prime}}$.

Since if $P(x) \leftarrow R_{\text {row }(s)}(x)$ for some $s$ in $S$, then $D(M)(s)=1$, and since $\phi(\operatorname{row}(s))$ is mapped to a state $q$ with $\operatorname{row}(q)=\operatorname{row}(s)$, it must be that $q$ is in $F$ and hence $P(x) \leftarrow R_{q}(x)$. Conversely, if $\operatorname{row}(s)$ is mapped to a state $q$ such that $P(x) \leftarrow R_{q}(x)$ is in 
$\mathrm{LMLP}_{\mathrm{A}}$, then since $\mathrm{q}$ is in $\mathrm{F}$ and $\operatorname{row}(\mathrm{q})=\operatorname{row}(\mathrm{s}), \mathrm{D}(\mathrm{M})(\mathrm{s})=1$, so $\mathrm{P}(\mathrm{x}) \leftarrow \mathrm{R}_{\text {row }(\mathrm{s})}(\mathrm{x})$ is in LMLP $_{M}$. So we conclude that the mapping $\phi$ preserves the clauses. Q.E.D.

\section{Inductive inference algorithm for linear monadic logic programs}

First we confirm the inductive inference schema of linear monadic logic programs. The problem is to identify the denotation of the predicate $P$ in the unknown model. That is, in our setting the problem is to infer a linear monadic logic program LMLP such that the denotation of $P$ in $\cap M(L M L P)$ is equal to the one in the unknown model.

Let the unknown model for some linear monadic logic program be denoted by $M_{U}$.

\section{(Algorithm IL of inductive inference for linear monadic logic programs)}

Input : An oracle $\mathrm{EX}($ ) for a sufficient set of examples (or facts of ground atoms) of the predicate $P$ in $M_{U}$,

An oracle MEMBER(P(t)) on a ground atom $P(t)$ as input for a membership query. to output 1 or 0 according to whether $P(t)$ is true in $M_{U}$,

Output: A sequence of conjectures of linear monadic logic program,

Procedure :

$\mathrm{S}:=\varnothing ; \mathrm{E}:=\{\$\} ; \mathrm{LMLP}:=\varnothing ;$ Examples $:=\varnothing ;$

do forever

add an example EX() to Examples;

while there is a negative example $-P(t) \in$ Examples such that LMLP $\vdash P(t)$

or there is a positive example $+\mathrm{P}(\mathrm{t}) \in$ Examples such that LMLP $H \mathrm{P}(\mathrm{t})$;

add $t$ and all its subtrees to $S$;

extend (S, E, M) to E.(SUX(S)) using MEMBER;

repeat

if $(\mathrm{S}, \mathrm{E}, \mathrm{M})$ is not consistent

then find $s_{1}$ and $s_{2}$ in $S, f \in \Gamma_{n}, u_{1}, \ldots, u_{n-1} \in S, e \in E$, and $i(1 \leqq i \leqq n)$ such that

row $\left(s_{1}\right)$ is equal to row $\left(s_{2}\right)$ and $D(M)\left(e \cdot f\left(u_{1}, \ldots, u_{i-1}, s_{1}, u_{i}, \ldots, u_{n-1}\right)\right)$

$\neq D(M)\left(e \cdot f\left(u_{1}, \ldots, u_{i-1}, s_{2}, u_{i}, \ldots, u_{n-1}\right)\right) ;$

add $e \cdot f\left(u_{1}, \ldots, u_{i-1}, \$, u_{i}, \ldots, u_{n-1}\right)$ to $E$;

extend (S, E, M) to E.(SUX(S)) using MEMBER;

if $(S, E, M)$ is not closed;

then find $f(\bar{u}) \in X(S)$ for $\bar{u} \in S^{n}$ and $f \in \Gamma_{n}$ such that row $(f(\bar{u}))$ is different

from row $(s)$ for all $s \in S$;

add $f(\bar{u})$ to $S$; 
extend (S, E, M) to E.(SUX(S)) using MEMBER;

until (S, E, M) is closed and consistent;

LMLP : = LMLP $_{M}$;

end;

output LMLP;

end.

In the above algorithm, the operation of "extend (S, E, M) to E.(SUX(S)) using MEMBER" is the operation to extend $D(M)$ by asking membership queries for missing elements. We call an example $t$ presented by the oracle EX a counterexample when the last conjecture LMLP ${ }_{M}$ does not agree with $t$.

Example 3 Suppose the unknown linear monadic logic program is the one of example 2. Then the algorithm IL identifies the following linear monadic logic program from 2 examples $\{+t,-u\}$ after asking 23 membership queries.

(Predicate characterization matrix)

“E"

\begin{tabular}{|c|c|c|}
\hline $\mathrm{M}$ & $\$$ & $\neg(\$)$ \\
\hline $\mathrm{t}$ & 1 & 0 \\
\hline$\neg \mathrm{t}$ & 0 & 1 \\
\hline $\mathrm{u}$ & 0 & 0 \\
\hline $\mathrm{t} \vee \mathrm{t}$ & 1 & 0 \\
\hline$\neg(\neg \mathrm{t})$ & 1 & 0 \\
\hline$(\neg \mathrm{t}) \vee \mathrm{t}$ & 1 & 0 \\
\hline$t \vee(\neg \mathrm{t})$ & 1 & 0 \\
\hline$(\neg \mathrm{t}) \vee(\neg \mathrm{t})$ & 0 & 1 \\
\hline$\neg \mathrm{u}$ & 0 & 0 \\
\hline $\mathrm{u} \vee \mathrm{t}$ & 1 & 0 \\
\hline $\mathrm{u} \vee(\neg \mathrm{t})$ & 0 & 0 \\
\hline $\mathrm{t} \vee \mathrm{u}$ & 1 & 0 \\
\hline$(\neg \mathrm{t}) \vee \mathrm{u}$ & 0 & 0 \\
\hline $\mathrm{u} \vee \mathrm{u}$ & 0 & 0 \\
\hline
\end{tabular}

(The identified linear monadic logic program) 


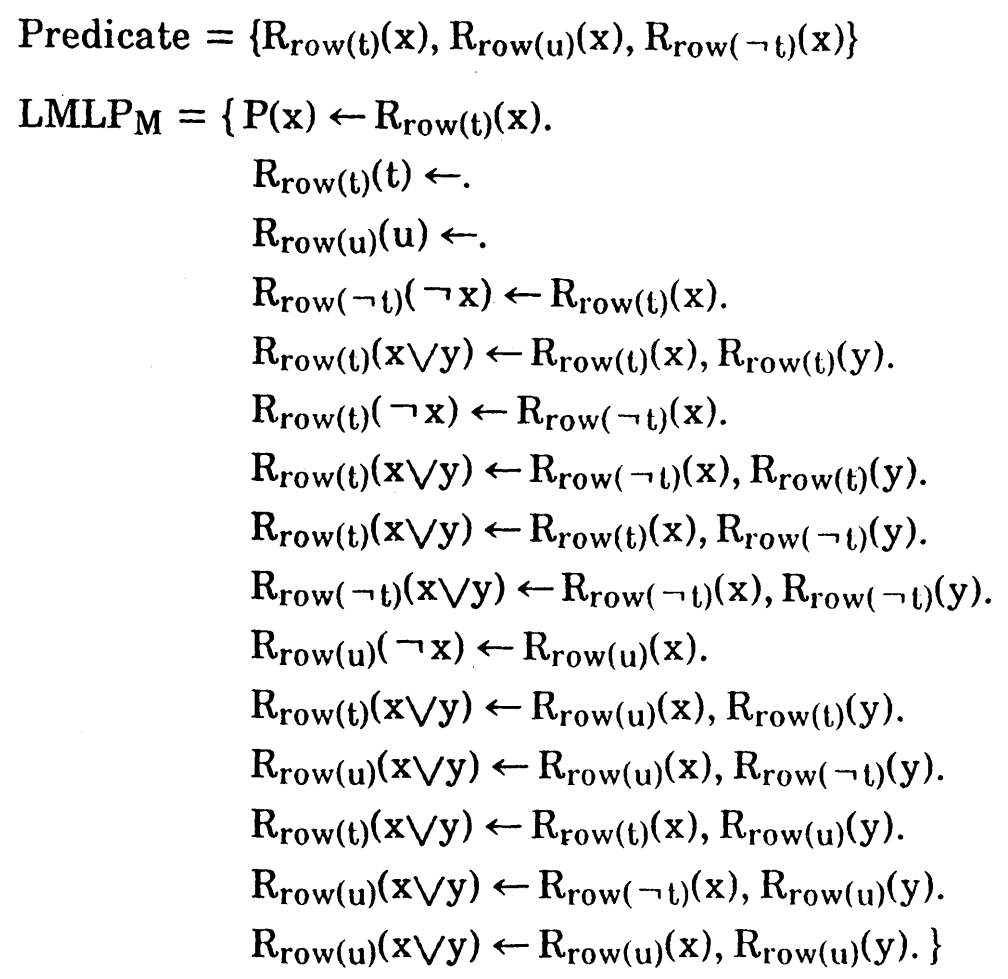

\section{Correctness and complexity}

To see that the algorithm IL is correct, i.e. the algorithm IL identifies a linear monadic logic program LMLP in the limit such that $\{t: P(t) \in \cap M(L M L P)\}$ is the denotation of $\mathbf{P}$ by $\mathrm{M}_{U}$, it is enough for us to show that the constructed predicate characterization matrix $(\mathrm{S}, \mathrm{E}, \mathrm{M})$ during the running of the algorithm IL is a closed, consistent one such that $\mathrm{S}$ is subtree-closed and $\mathrm{E}$ is $\$$-prefix-closed with respect to $\mathrm{S}$, and that the while loop of the algorithm IL is executed at most in finite time during the running of the algorithm IL.

Lemma 6.1 Let (S, E, M) be a predicate characterization matrix such that $\mathrm{S}$ is subtree-closed and $\mathrm{E}$ is \$-prefix-closed with respect to $\mathrm{S}$. Let $n$ be the number of different values of row(s) for $\mathrm{s}$ in $\mathrm{S}$. Any deterministic tree automaton which agrees with the data in $\mathrm{M}$ must have at least $n$ states.

(Proof) Let $\mathrm{T}_{\mathrm{A}}=(\mathrm{Q}, \Gamma, \delta, \mathrm{F})$ be a deterministic tree automaton which agrees with the data in $M$. Suppose that $s_{1}$ and $s_{2}$ are elements of $S$ such that row $\left(s_{1}\right)$ and row $\left(s_{2}\right)$ are distinct. Then there exists e in $E$ such that $D(M)\left(e \cdot s_{1}\right) \neq D(M)\left(e \cdot s_{2}\right)$. Since $T_{A}$ agrees with the data in $M$, exactly one of $\delta\left(e \cdot s_{1}\right)$ and $\delta\left(e \cdot s_{2}\right)$ is in $F$. Thus $\delta\left(s_{1}\right)$ and $\delta\left(s_{2}\right)$ must 
be distinct states because $\mathrm{T}_{\mathrm{A}}$ is deterministic. Since $\delta(\mathrm{s})$ takes on at least $\mathrm{n}$ different values as s ranges over $\mathrm{S}, \mathrm{T}_{\mathrm{A}}$ must have at least $\mathrm{n}$ states. Q.E.D.

Lemma 6.2 The while loop of the algorithm IL is executed at most in finite time during the running of the algorithm $\mathrm{IL}$.

(Proof) Let $\mathrm{n}$ be the number of states in the minimum state deterministic tree automaton $\mathrm{T}_{\mathrm{A}}$ for the denotation of the predicate $\mathrm{P}$ in the unknown model. Firstly we will show that whenever a predicate characterization matrix $(\mathrm{S}, \mathrm{E}, \mathrm{M})$ is not consistent or not closed, the number of distinct values row(s) for s in S must increase. If $(\mathrm{S}, \mathrm{E}, \mathrm{M})$ is not consistent, then since two previously equal row values, row $\left(\mathrm{s}_{1}\right)$ and row $\left(\mathrm{s}_{2}\right)$, are no longer equal after $\mathrm{E}$ is augmented, the number of distinct values row(s) for $\mathrm{s}$ in $\mathrm{S}$ must increase by at least one. If $(\mathrm{S}, \mathrm{E}, \mathrm{M})$ is not closed and a tree $\mathrm{f}(\overline{\mathrm{u}})$ is added to $S$, then since $\operatorname{row}(f(\bar{u}))$ is different from row(s) for all $s$ in $S$ before $S$ is augmented, the number of distinct values row (s) must increase by at least one.

Next we will show that whenever a tree $t$ and all its subtrees are added to $S$ and $(\mathrm{S}, \mathrm{E}, \mathrm{M})$ is extended because $\mathrm{LMLP}_{M}$ does not agree with $\mathrm{t}$, the extended closed, consistent predicate characterization matrix (S', E', M') have at least one more different row values than (S, E, M). Assume that (S, E, M) and (S', E', M') have the same number of different row values. Then both must have the same row values. Since (S', E', M') is closed and consistent, each of t and all its subtrees plays the same role in ( $\left.S^{\prime}, E^{\prime}, M^{\prime}\right)$ as an element $s$ in $S \subseteq S^{\prime}$ which has the same row value as it. Hence from (S, E, M) and from (S', E', M'), a same linear monadic logic program is constructed, i.e. $L M L P_{M}=L M L P_{M}$. However, by theorem 4.4, $L M L P_{M}$ ' agrees with $t$ while $L M L P_{M}$ does not agree with $t$. This is a contradiction.

Then by these and lemma 6.1 and theorem 4.5, (S, E, M) can be not consistent or not closed at most $n-1$ times and a counter-example is added to $S$ at most $n$ times during the running of the algorithm IL. Thus whenever the condition of the while loop becomes true, the algorithm IL eventually makes a next conjecture in finite 
time, and the condition of the while loop becomes true at most $\mathrm{n}$ times. Therefore, the while loop is executed at most in finite time. Q.E.D.

By the above result, it follows that the algorithm IL makes a sequence of at most n conjectures.

Lemma 6.3 The conjectures which the algorithm IL makes are correct for the facts known by the oracles EX and MEMBER.

(Proof) We will show that each predicate characterization matrix (S, E, M) which the algorithm IL constructs during the running of it is a closed, consistent one such that $\mathrm{S}$ is subtree-closed and $\mathrm{E}$ is $\$$-prefix-closed with respect to $\mathrm{S}$. In the algorithm IL, there are three operations which extend the row or the column of $(\mathrm{S}, \mathrm{E}, \mathrm{M})$. When $t$ and all its subtrees are added to $S, S$ obviously remains subtree-closed. If (S, E, M) is not consistent, then for some $f \in \Gamma_{n}, u_{1}, \ldots, u_{n-1} \in S, e \in E$, and $i(1 \leqq i \leqq n)$, e. $f\left(u_{1}, \ldots, u_{i-1}, \$, u_{i}, \ldots, u_{n-1}\right)$ is added to $E$. In this case, E remains $\$$-prefix-closed with respect to $S$. If ( $S, E, M)$ is not closed, then for some $\bar{u} \in S^{n}$ and $f \in \Gamma_{n}, f(\bar{u})$ is added to $S$. In this case, $\mathrm{S}$ remains subtree-closed. Since the repeat loop is repeated as long as $(\mathrm{S}$, $\mathrm{E}, \mathrm{M}$ ) is not closed and consistent, by lemma 6.2 , each constructed (S, E, M) must eventually be closed and consistent. 'Thus each constructed (S, E, M) during the running of the algorithm IL is a closed, consistent one such that $\mathrm{S}$ is subtree-closed and $\mathrm{E}$ is $\$$-prefix-closed with respect to $\mathrm{S}$. 'Then by theorem 4.4 , the conjectures of linear monadic logic program which the algorithm IL makes are correct for the facts known by the oracles EX and MEMBER. Q.E.D.

Now we conclude the following theorem.

Theorem 6.4 The algorithm IL identifies in the limit a linear monadic logic program LMLP such that $\{\mathrm{t}: \mathrm{P}(\mathrm{t}) \in \cap \mathrm{M}(\mathrm{LMLP})\}$ is equal to the denotation of $\mathrm{P}$ in $\mathrm{M}_{\mathrm{U}}$.

Next we will analyse the time complexity of the algorithm IL. By lemma 6.2, the while loop of the algorithm IL is executed at most in a finite time. Then how much 
time does the while loop consume during the running of the algorithm IL. 'That depends partly on the size of the examples presented by the oracle EX. We will analyze the running time of the while loop as a function of $n$, the number of states in the minimum tree automaton for the denotation of the predicate $\mathrm{P}$ in the unknown model, and $\mathrm{m}$, the maximum size of any counter-examples presented by EX during the running of the algorithm $\mathrm{IL}$, where the size of an example is the number of symbols in its textual representation. We will show that its running time is bounded by a polynomial in $\mathrm{m}$ and $\mathrm{n}$. Let $\mathrm{k}$ be the cardinality of the alphabet $\Gamma$ and $\mathrm{d}$ be the maximum arity of the function symbols in $\Gamma$. We may assume $d \geq 1$.

Whenever (S, E, M) is discovered to be not closed, one element is added to $\mathrm{S}$. Whenever ( $\mathrm{S}, \mathrm{E}, \mathrm{M})$ is discovered to be not consistent, one element is added to $\mathrm{E}$. For each counter-example $\mathrm{t}$ of size at most $\mathrm{m}$ presented by the oracle $\mathrm{EX}$, at most $\mathrm{m}$ subtrees are added to $\mathrm{S}$. Since the predicate characterization matrix is discovered to be not consistent at most $n-1$ times, the total number of trees in $E$ cannot exceed $n$. Since the predicate characterization matrix is discovered to be not closed at most $\mathrm{n}-1$ times, and there can be at most $\mathrm{n}$ counter-examples, the total number of trees in $S$ cannot exceed $n+m n$. Thus, the maximum cardinality of $E \cdot(S \cup X(S))$ is at most

$$
n\left((n+m n)+k(n+m n)^{d}\right)=O\left(m^{d} n^{d+1}\right) .
$$

Now we consider the operations in the while loop executed by the algorithm IL. Checking the predicate characterization matrix to be closed and consistent can be done in time polynomial in the size of the matrix and must be done at most $\mathrm{n}$ times. Adding a tree to $S$ or $E$ requires at most $O\left(m^{d} n^{d}\right)$ membership queries to extend the matrix. When the predicate characterization matrix is closed and consistent, LMLP $_{M}$ may be constructed in time polynomial in the size of the matrix, and this must be done at most $\mathrm{n}$ times. A counter-example requires the addition of at most $\mathrm{m}$ subtrees to $\mathrm{S}$, and this can be also happen at most $\mathrm{n}$ times.

Therefore, the total time which the while loop consumes during the running of the algorithm IL can be bounded by a polynomial function of $\mathrm{m}$ and $\mathrm{n}$. 
On the other hand, the check whether a conjecture agrees with an example, i.e. LMLPト $P(t)$ or not, in the condition of the while loop is decidable and is performed in steps of the example's size. Then by the above result, we can conclude that the algorithm IL infers a conjecture of a linear monadic logic program consistently and requests a new example in time polynomial in $1, \mathrm{~m}^{\prime}$ and $\mathrm{n}$ after the last example has been added, where 1 is the number of examples known at the time of the request and $m$ ' is the maximum size of those 1 known examples.

\section{Concluding remarks}

We remark on related work. Shapiro's Model Inference System (MIS for short) $[5,6]$ is the excellent and only existing system to infer logic programs or Herbrand models in first order logic using the concept of identification in the limit defined by Gold. MIS can infer a large class of logic programs (h-easy models), but ours only for a restricted class of logic programs. However our algorithm IL has several unique features compared with MIS. (1) As we mentioned in the introduction, our algorithm IL is based on algebraic semantics and the target of the inference is a tree language computed by a logic program, and hence it is different from Shapiro's approach and is not model inference. (2) In general, it is not easy to analyse the time complexity of inductive inference algorithm, and neither in MIS. We have shown in the last section the time complexity of our algorithm IL in the very clear manner. (3) Our algorithm IL is based on the constructive method, while MIS is based on the enumerative method, where the constructive method systematically use examples to construct the conjecture and the enumerative method use them to select a conjecture in enumeration. It is said that the constructive method is in general more efficient than the enumerative method. (4) In our algorithm IL, the predicate symbol P and its interpretation are only given as the observational language and the oracle, and any information about the hypothesis language is not given. The algorithm IL automatically generates other predicates whenever they are needed. However in MIS, all predicates used to construct the conjectures and those intended 
interpretations must also be given as the hypothesis language and the oracle, and this is often referred to as the problem about theoretical terms of MIS.

\section{Acknowledgements}

The author would like to thank Dr. T.Kitagawa, the president of IIAS-SIS, Dr. H.Enomoto, the director of IIAS-SIS, for giving him the opportunity to pursue this work and helping him. He is deeply grateful to Dr. T.Yokomori for reading the draft and giving him many valuable comments. Discussions with the colleagues Y.Takada and H.Ishizaka were also very fruitful.

This is part of the work in the major R\&D of the Fifth Generation Computer Project, conducted under program set up by MITI.

\section{References}

[1] Angluin,D., Learning regular sets from queries and counter-examples, Yale DCS $T R-464,1986$. To appear in Information and Computation.

[2] van Emden,M.H., Kowalski,R.A., The semantics of predicate logic as a programming language, J. ACM 23 (1976), 733-742.

[3] Gold,E.M., Complexity of automaton identification from given data, Information and Control 37 (1978), 302-320.

[4] Marque-Pucheu,G., Rational set of trees and the algebraic semantics of logic programming, Acta Informatica 20 (1983), 249-260.

[5] Shapiro,E., Algorithmic program debugging, MI'T Presc, 1983.

[6] Shapiro,E., Inductive inference of theories from facts, Yale DCS TR-192, 1981. 